\title{
A pneumonia that will not go away
}

\author{
Khaled R Khasawneh MD', Tashfeen Mahmood MD², Ruba A Halloush MD³, Faisal A Khasawneh MD
}

KR Khasawneh, T Mahmood, RA Halloush, FA Khasawneh. A pneumonia that will not go away. Can Respir J 2014;21(2):80-82.

Pneumonia is a common diagnosis with significant morbidity and mortality. However, pneumonia is a commonly overdiagnosed entity, with many similar-appearing conditions. A young, previously healthy woman was misdiagnosed with a variety of respiratory tract infections over the course of five months before establishing the correct diagnosis - chronic eosinophilic pneumonia.

Key Words: Chronic respiratory symptoms; Eosinophilia; Eosinophilic pneumonia

Learning objectives

1. To recognize the presenting symptoms of chronic eosinophilic pneumonia (CEP).

2. To understand the management of CEP.

CanMEDs Competency: Medical Expert

Pretest

- What are the diagnostic criteria of CEP?

- How would you treat a patient who presents with CEP?

\section{CASE PRESENTATION}

A 28-year-old woman was admitted with cough and few days' history of progressive shortness of breath and fevers. Her illness started five months previously when she was hospitalized with similar symptoms and was diagnosed with a community-acquired pneumonia. Since that admission, she was diagnosed with bronchitis twice and was treated as an outpatient. Between her acute illnesses, she continued to report dry irritating cough and dyspnea. She denied heartburn symptoms or history of sinusitis. Her medical history was negative and she had no history of smoking. She lived on a ranch in rural West Texas (USA), where she tended cows, goats, chicken and dogs. She was married with two children and none of her family members reported similar symptoms.

On examination, the patient was a healthy-appearing female in no respiratory distress. Vital signs were as follows: temperature $37.8^{\circ} \mathrm{C}$; heart rate 95 beats/min (regular); blood pressure 101/58 $\mathrm{mmHg}$; respiratory rate 22 breaths/min; and oxygen saturation $93 \%$ while on $2 \mathrm{~L} / \mathrm{min}$ of oxygen via nasal cannula. Significant physical examination findings included the following; chest, wheezing and prolonged expiration; regular heart sounds without murmurs or gallop and no lymphadenopathy or skin rashes.

Pertinent laboratory findings included the following: white blood cell count $4.4 \times 10^{9} / \mathrm{L}$ (73\% neutrophils, $20 \%$ lymphocytes and $5 \%$ eosinophils); hemoglobin level $129 \mathrm{~g} / \mathrm{L}$; platelet count $121 \times 10^{9} / \mathrm{L}$; procalcitonin level $0.2 \mathrm{ng} / \mathrm{mL}$, C-reactive protein level $25.8 \mathrm{mg} / \mathrm{L}$, erythrocyte sedimentation rate $16 \mathrm{~mm} / \mathrm{h}$, antinuclear antibodies and rheumatoid factor testing was negative and complement levels were within normal limits; airway cultures and blood cultures were negative;

\section{Une pneumonie persistante}

La pneumonie est un diagnostic courant qui s'accompagne d'une morbidité et d'une mortalité importantes. Cependant, elle est souvent surdiagnostiquée, car de nombreuses infections lui ressemblent. Sur une période de cinq mois, une jeune femme auparavant en santé a reçu divers diagnostics erronés d'infections respiratoires avant que le bon diagnostic de pneumonie à éosinophiles chronique soit posé.

HIV test and serologies for Q fever and brucella were negative. Renal function, electrolytes, liver enzyme levels and coagulation studies were within normal limits. Pulmonary function testing (PFT) revealed a forced expiratory volume in $1 \mathrm{~s}\left(\mathrm{FEV}_{1}\right) 45 \%$ of predicted, forced vital capacity (FVC) $55 \%$ of predicted and $\mathrm{FEV}_{1} / \mathrm{FVC}$ ratio of 64 . There was a positive bronchodilator response documented by an increase in FVC by $230 \mathrm{~mL}$ after the patient received albuterol. Her total lung capacity was $70 \%$ of predicted, with a diffusing capacity of carbon monoxide (DLCO) $74 \%$ of predicted.

A chest radiograph (CXR) revealed prominent interstitial markings (Figure 1). A computed tomography scan of the chest revealed nonspecific ground-glass opacities (Figure 2). Given the relapsing nature of her complaints and the absence of a definite infectious etiology, an open-lung biopsy (OLB) was performed. The scant and peripherally distributed pulmonary ground-glass opacities, in addition to the patient's low surgical risk supported an OLB as opposed to transbronchial biopsy, and was well tolerated.

The OLB revealed peribronchiolar inflammatory infiltrate composed predominantly of eosinophils in addition to some lymphocytes and plasma cells (Figure 3). No acute inflammation in alveolar spaces, viral changes, foreign bodies, vasculitis or granulomas were identified. The patient was diagnosed with CEP. Empirical antibiotic therapy was discontinued and systemic steroids were started. She was seen in clinic one month after discharge and was completely asymptomatic; a slow, gradual steroid taper was initiated.

\section{DISCUSSION}

Eosinophilic lung disorders are a heterogeneous group of pulmonary diseases characterized by the presence of blood and/or lung eosinophilia (1). These ailments can result from a primary eosinophilic lung process including simple pulmonary eosinophilia, acute eosinophilic pneumonia and CEP, Churg-Strauss vasculitis and allergic bronchopulmonary aspergillosis, or a secondary illness caused by drugs, parasites and/or fungi. Thorough history taking including travel and environmental exposures, physical examination, appropriate imaging, PFT and, occasionally, tissue sampling, is essential in establishing a specific diagnosis and determining therapy.

CEP is a rare disease with unknown etiology and insidious onset (2). Patients present with subacute or chronic respiratory symptoms including cough, dyspnea fever and weight loss. The disease is more common in women, with a ratio of 2:1. Most patients are nonsmokers in their third and fourth decades of life $(2,3)$.

\footnotetext{
${ }^{1}$ Division of Pulmonary and Critical Care Medicine, University of Arkansas for Medical Sciences, Little Rock, Arkansas; ${ }^{2}$ Department of Internal Medicine, Deaconess Hospital, Evansville, Indiana; ${ }^{3}$ Amarillo Pathology Group, Amarillo; ${ }^{4}$ Section of Critical Care, Department of Internal Medicine, Texas Tech University Health Sciences Center, Amarillo, Texas, USA

Correspondence: Dr Faisal A Khasawneh, Section of Critical Care Medicine, Department of Internal of Medicine, Texas Tech University Health Sciences Center, 1400 South Coulter Street, Amarillo, Texas 79106, USA. Telephone 806-354-5480, fax 806-354-5765,

e-mail faisal.khasawneh@ttuhsc.edu
} 


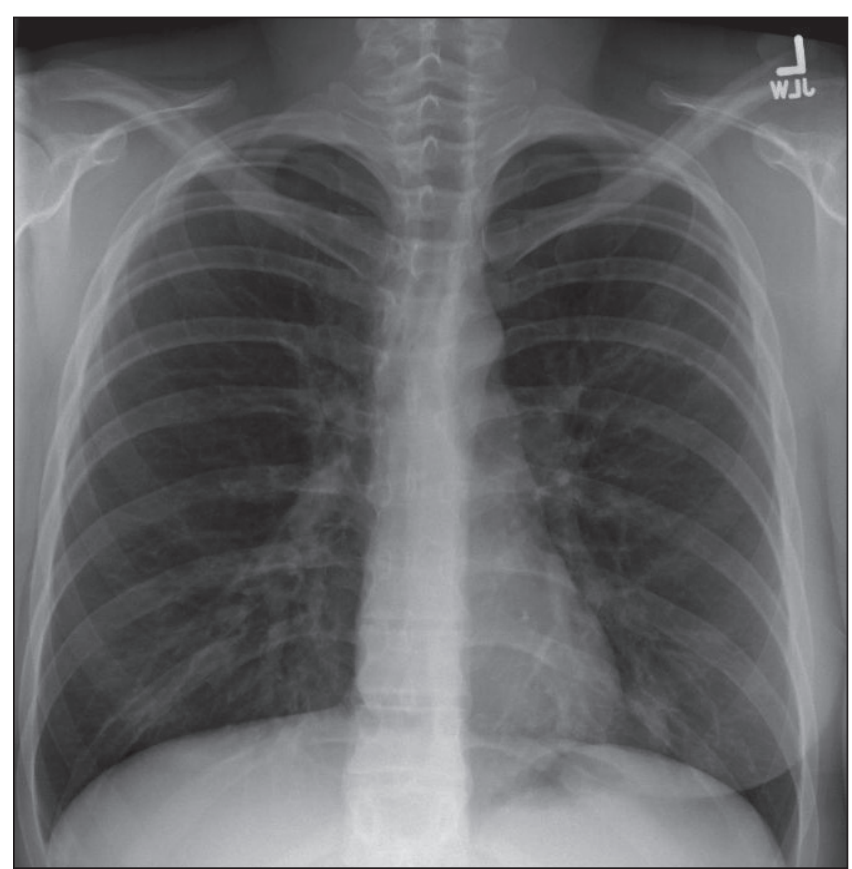

Figure 1) Chest radiograph showing prominent bilateral lower lobe interstitial pulmonary markings

The majority of patients have peripheral blood eosinophilia and an elevated erythrocyte sedimentation rate (3). Iummnoglobulin E levels are elevated in $50 \%$ of patients. PFT can be normal, obstructive or restrictive, and DLCO is usually reduced. Peripheral parenchymal infiltrates are common on CXR and mimic the photographic negative of acute pulmonary edema (4). Computed tomography of the chest will confirm the parenchymal opacities but may reveal ground-glass opacities that are not apparent on CXR. Pleural effusions are rare; however, the typical pattern is not present in the majority of patients and are apparent in other interstitial lung diseases (4). The percentage of eosinophils is elevated in bronchoalveolar lavage (BAL) fluid. Lung biopsy shows eosinophilic infiltration of intra-alveolar septa and alveolar spaces.

Oral corticosteroids are the mainstay of therapy for CEP (3). The clinical response is rapid and most patients will experience complete resolution of their symptoms. The usual dose of prednisone is between $0.5 \mathrm{mg} / \mathrm{kg} /$ day and $1 \mathrm{mg} / \mathrm{kg} / \mathrm{day}$ followed by gradual tapering over a sixto 12 -month period (3). Relapse of CEP is very common and some patients will require long-term maintenance therapy with steroids.

In contrast to the above, acute eosinophilic pneumonia presents with rapid-onset respiratory failure that may require intubation and mechanical ventilation. Patients present with fever, myalgia and shortness of breath accompanied by severe hypoxemia and bilateral lung airspace opacities with rapid progression to severe respiratory failure (5). In contrast to CEP, there is no peripheral eosinophilia, but the percentage of eosinophils in BAL is elevated (5). There is rapid and dramatic clinical and radiographic response to corticosteroids. Most patients remain disease free with no recurrence after the discontinuation of corticosteroids.

Simple pulmonary eosinophilia is a self-limiting disease that presents with mild respiratory symptoms, migratory lung opacities and peripheral eosinophilia. Parasitic and fungal infections as well as drug reactions should be ruled out before diagnosing this entity (1).

Churg-Strauss syndrome is a systemic vasculitis that commonly develops in the setting of antecedent asthma, allergic rhinitis or sinusitis. Patients usually present with peripheral eosinophilia, migratory pulmonary opacities, purpuric rash, fever and arthralgia (6). Complaints related to cardiac, gastrointestinal or renal involvement are less common. P-ANCA is positive in $40 \%$ of patients (6). Tissue biopsy establishes the diagnosis (6). Most patients respond quickly to
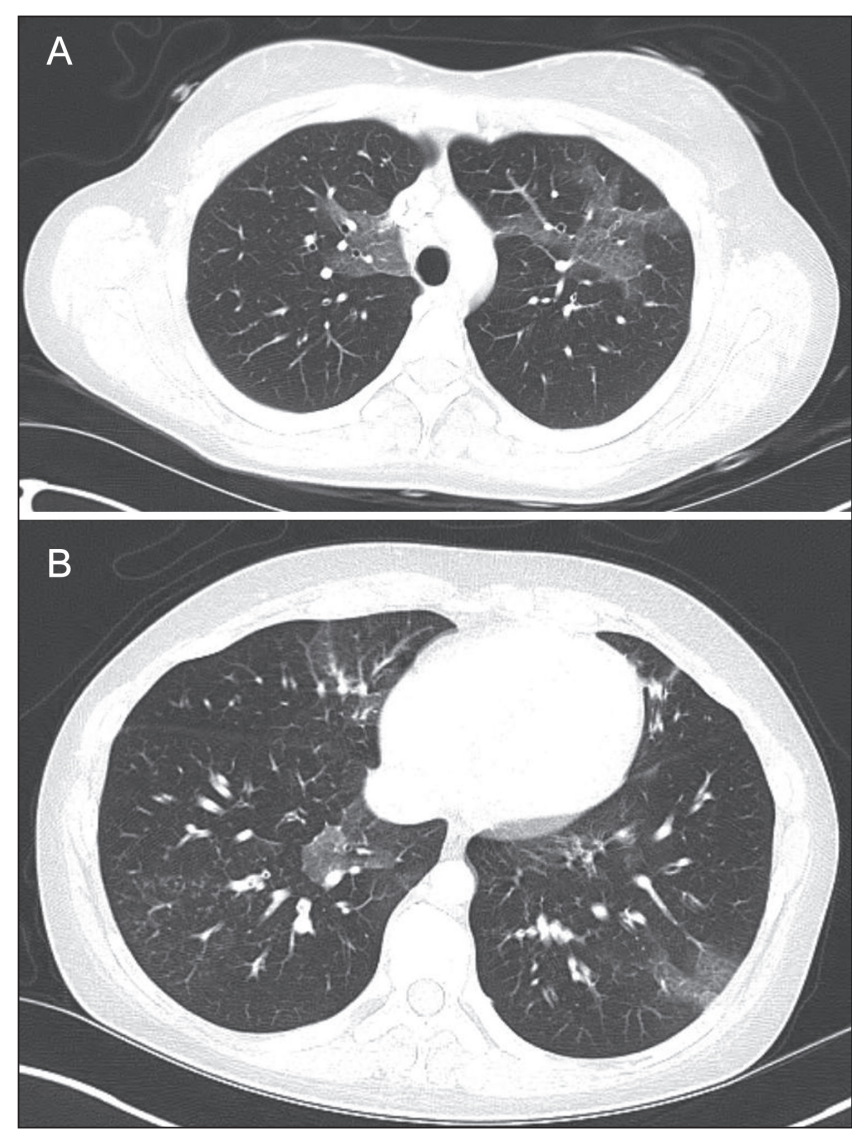

Figure 2) A and B Chest computed tomography scans demonstrating scattered ground-glass pulmonary opacities without plerual effusions or lymphadenopathy

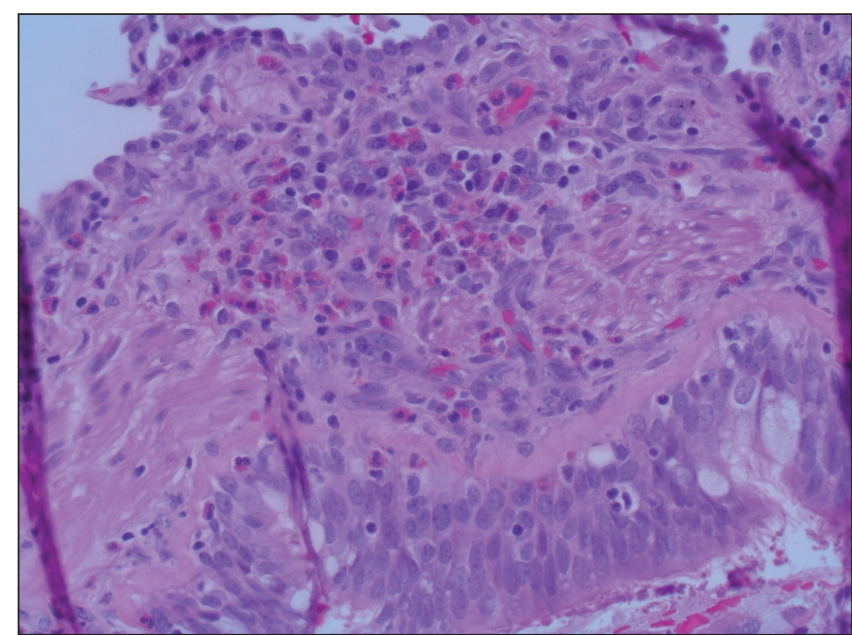

Figure 3) Lung wedge biopsy showing peribronchiolar eosinophilic inflammatory infiltrate (hematoxylin and eosin stain, original magnification $\times 400$ )

high-dose steroids. Severe cases may require cyclophosamide. Relapses are frequent; therefore, suppressive therapy with azathioprine or methotrexate for 12 to 18 months is recommended (6).

Allergic bronchopulmonary aspergillosis is a hypersensitivity lung disease associated with inflammatory destruction of airways in response to Aspergillus species. This disease's diagnostic criteria include: asthma, central bronchiectasis, Aspergillus fumigatus hyper-reactivity documented by elevated specific immunoglobulin levels or, on skin testing, fleeting pulmonary opacities and peripheral eosinophilia (7). Corticosteroids are a cornerstone of therapy; itraconazole has a demonstrable steroid-sparing effect (7). 
Idiopathic hypereosinophilic syndrome is a rare myeloproliferative disorder characterized by peripheral eosinophilia that has been present for $>6$ months associated with evidence of end-organ damage caused by eosinophilic infiltration without any other identifiable etiology (1). Pulmonary manifestations include chronic cough, asthma and pulmonary fibrosis. Cardiac involvement is a main cause of mortality in patients with this disease (1).

Secondary eosinophilic lung diseases result from certain parasitic and fungal infections or the exposure to certain drugs. In North America, Strongyloides, Ascaris, Toxocara and Ancylostoma are the most common parasitic causes of eosinophilic lung disease $(8,9)$. In primary coccidioidomycosis, an endemic fungus in the southwestern corner of the United States, peripheral blood eosinophilia is noted in the majority of cases and pulmonary eosinophilic infiltrates may be demonstrated on lung biopsy or BAL examination (10). Drugs commonly reported to cause eosinophilic lung disease include amiodarone, cocaine, nonsteroidal anti-inflammatory drugs, penicillins and sulpha compounds (11).

\section{Post-test}

- What are the diagnostic criteria for CEP?

Patients with CEP present with chronic respiratory symptoms, peripheral blood eosinophilia, abnormal PFT and peripheral lung infiltrates. Diagnosing CEP requires the presence of a consistent clinical picture in addition to the identification of eosinophilic pulmonary infiltrate on bronchoscopy or lung biopsy, in combination with excluding other eosinophilic disorders, especially parasitic infections.

- How would you treat a patient who presents with CEP? Systemic corticosteroids are the mainstay therapy for CEP. The disease is steroid responsive but requires prolonged therapy. Given the high prevalence in women and the need for chronic corticosteroid therapy, special measures should be taken to prevent osteoporosis.
DISCLOSURES: The authors have no financial disclosures or conflicts of interest to declare.

\section{REFERENCES}

1. Allen JN, Davis WB. Eosinophilic lung diseases. Am J Respir Crit Care Med 1994;150(5 Pt 1):1423-38.

2. Jederlinic PJ, Sicilian L, Gaensler EA. Chronic eosinophilic pneumonia. A report of 19 cases and a review of the literature. Medicine (Baltimore) 1988;67:154-62.

3. Marchand E, Reynaud-Gaubert M, Lauque D, Durieu J, Tonnel AB, Cordier JF. Idiopathic chronic eosinophilic pneumonia.

A clinical and follow-up study of 62 cases. The Groupe d'Etudes et de Recherche sur les Maladies "Orphelines" Pulmonaires (GERM"O”P). Medicine (Baltimore) 1998;77:299-312.

4. Gaensler EA, Carrington CB. Peripheral opacities in chronic eosinophilic pneumonia: The photographic negative of pulmonary edema. AJR Am J Roentgenol 1977;128: 1-13.

5. Philit F, Etienne-Mastroïanni B, Parrot A, Guérin C, Robert D, Cordier JF. Idiopathic acute eosinophilic pneumonia: A study of 22 patients. Am J Respir Crit Care Med 2002;166:1235-9.

6. Guillevin L, Cohen P, Gayraud M, Lhote F, Jarrousse B, Casassus P. Churg-Strauss syndrome. Clinical study and long-term follow-up of 96 patients. Medicine (Baltimore) 1999;78:26-37.

7. Walsh TJ, Anaissie EJ, Denning DW, et al. Treatment of aspergillosis: Clinical practice guidelines of the Infectious Diseases Society of America. Clin Infect Dis 2008;46:327-60.

8. Roig J, Romeu J, Riera C, Texido A, Domingo C, Morera J. Acute eosinophilic pneumonia due to toxocariasis with bronchoalveolar lavage findings. Chest 1992;102:294-6.

9. Boggild AK, Keystone JS, Kain KC. Tropical pulmonary eosinophilia: A case series in a setting of nonendemicity. Clin Infect Dis 2004:39:1123-8.

10. Lombard CM, Tazelaar HD, Krasne DL. Pulmonary eosinophilia in coccidioidal infections. Chest 1987;91:734-6.

11. Solomon J, Schwarz M. Drug-, toxin-, and radiation therapyinduced eosinophilic pneumonia. Semin Respir Crit Care Med 2006;27:192-7. 


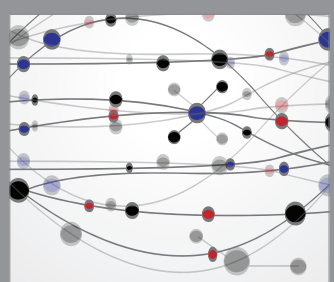

The Scientific World Journal
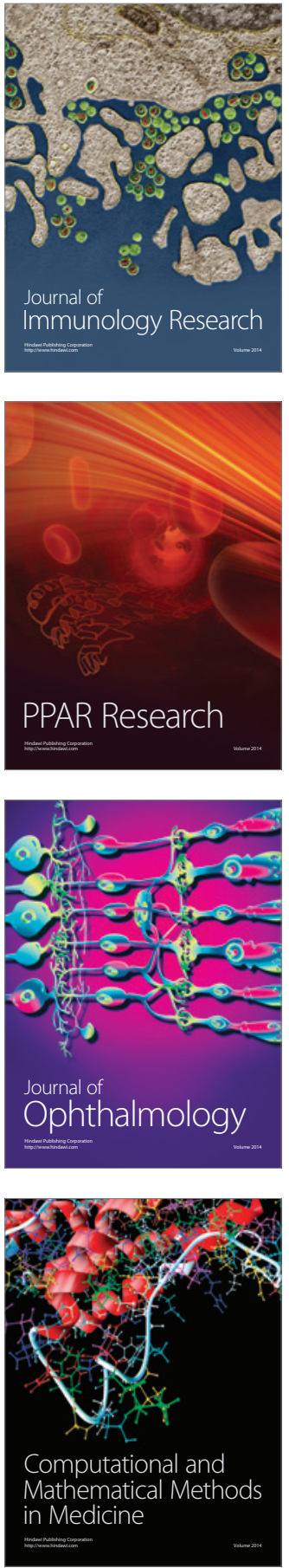

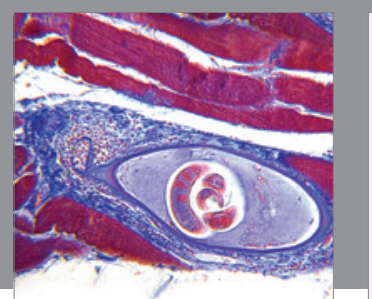

Gastroenterology Research and Practice

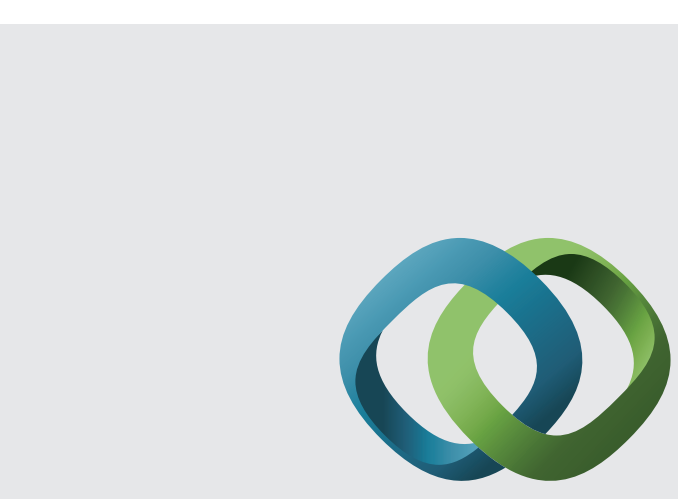

\section{Hindawi}

Submit your manuscripts at

http://www.hindawi.com
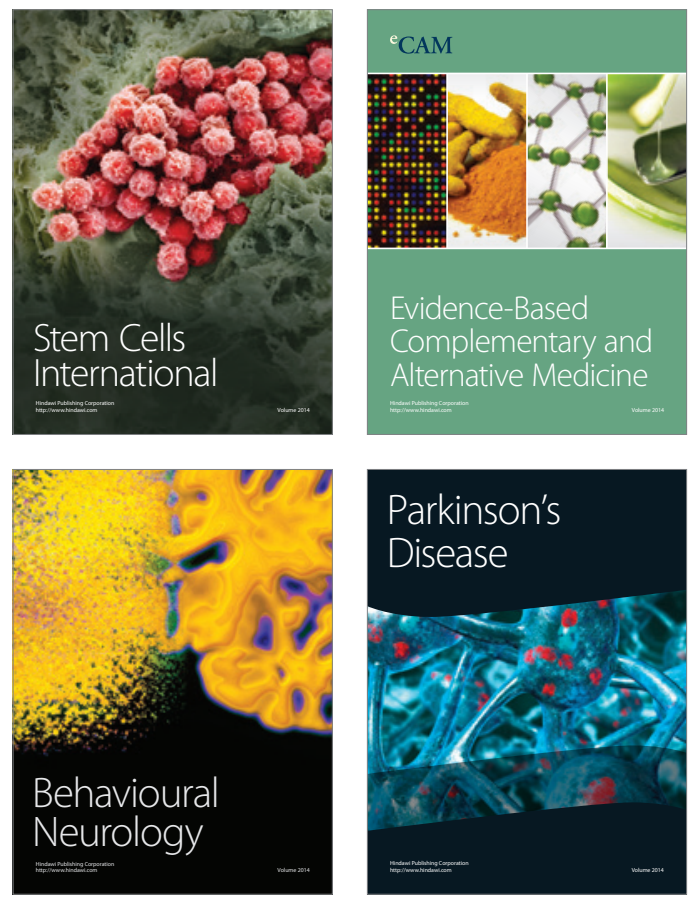
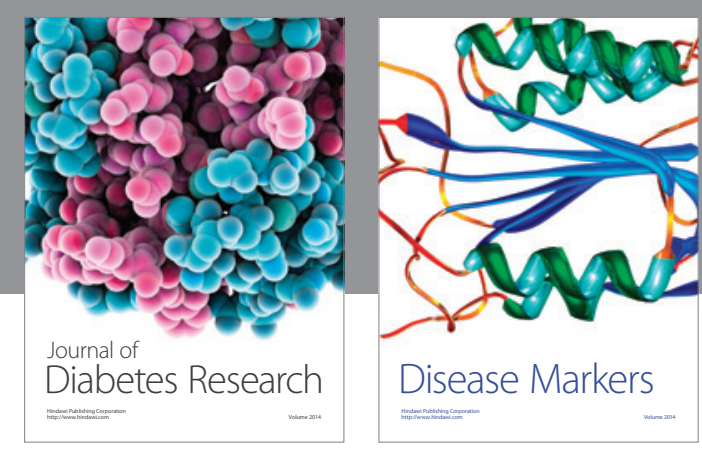

Disease Markers
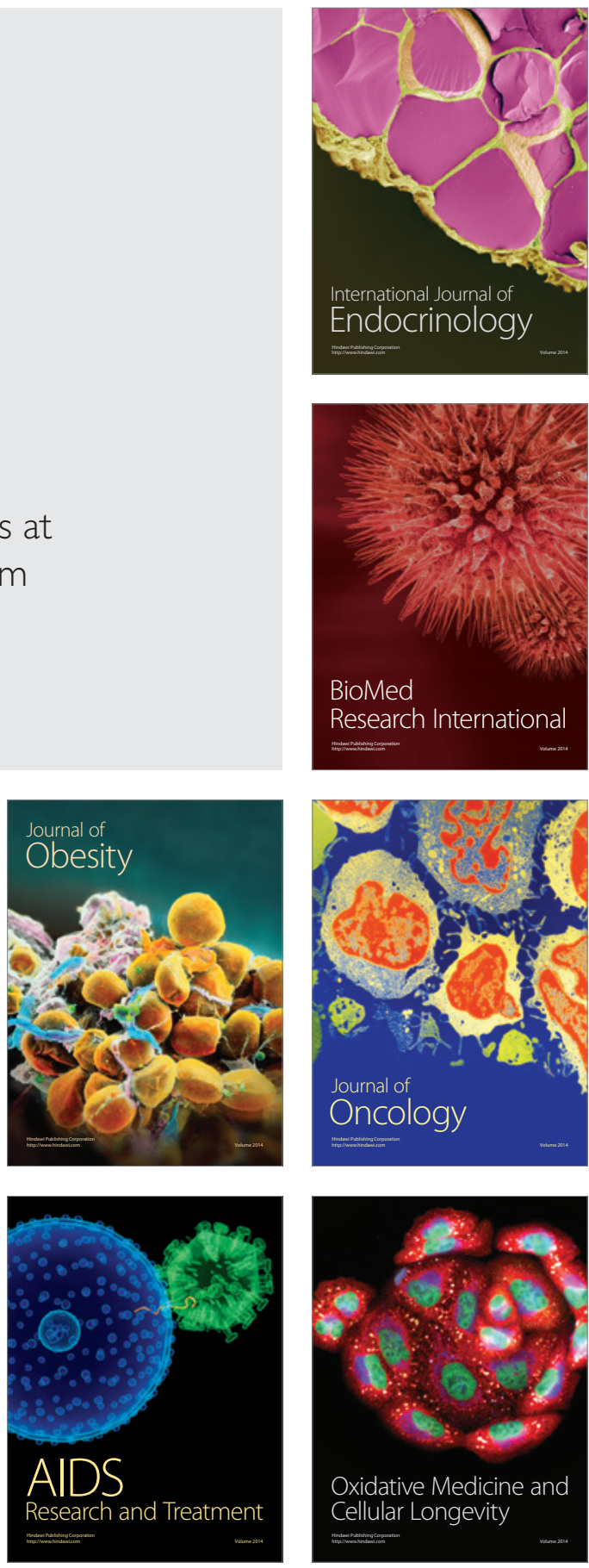